\title{
A Modified AC Current Controller for Active Loads
}

\author{
Abdullah Mahmoudi* and Seyed Hossein Hosseinian \\ Department of Electrical Engineering, Amirkabir University of Technology, Tehran, Iran; \\ abd_mahmoudi@aut.ac.ir, hosseinian@aut.ac.ir
}

\begin{abstract}
Active rectifier loads become more popular, recently, and their characteristics have been studied in the literature especially when they are connected to Micro Grids (MGs). In this paper, a modified AC current controller for such loads is proposed where the current direction and voltage drops of the output filter of active loads are involved in the controller. The proposed controller improves the performance of such loads either in single mode or in combined mode with MGs. Active load dynamics become relatively fast and smooth using the proposed method. The modified AC current controller makes the system more stable and also possible choices for controller parameters are widened. The validation and comparison of different case studies show the performance of the proposed method.
\end{abstract}

Keywords: Active Loads, AC Current Controller, Eigenvalues, Microgrids, Stability Analysis

\section{Introduction}

An electrical network may be contains either passive or active loads. Devices such as incandescent lighting or resistive space heaters are passive loads and they are typically modeled by a resistor or an inductor-resistor network. More relies on electronics by the population and also the penetrations of microgrid (MG) into power system ${ }^{1-4}$ have increased use of active loa ds, recently. A frontend rectifier which allows regulation of the voltage is used by many loads now. Devices such as machine drives, back-to-back converter configurations, and consumer electronics with unity power factor correction are active loads. The main characteristic of active loads is that they can be considered as constant power loads due to their regulated voltages over constant DC impedance loads. This characteristic is known to reduce damping and cause instabilities $^{5}$ and discussed in the literature ${ }^{5-14}$.

The modeling of active loads is presented using two methods. The first one neglects any dynamics from input filters or control loops of active load and assumes that it consists of only small-signal characteristics ${ }^{15}$. Using averaging methods, a linear state-space model for active loads is proposed in the second method considering the control and filter dynamics ${ }^{16}$. The second one is frequently used in the literature due to that dynamics of these loads are very important especially when they are connected to $\mathrm{MGs}^{17-18}$. The rectifier switching block is represented as an ideal transformer to use this assumption that the AC side power is equal to the DC side power ${ }^{17-18}$.

PWM rectifier using different current control structures is presented ${ }^{19}$ where different PI current controllers are investigated and their advantages are expressed. The filter of an active load may be constructed either by $\mathrm{LC}^{20}$ or LCL filters ${ }^{17-18}$. A superposition method is used to construct a simpler model for an active load ${ }^{21}$.

In this paper, a modified AC current controller for active loads based on the model proposed by N. Bottrell et al. ${ }^{17-18}$ is suggested. The performance of active loads either in single mode or in combined mode with MGs is improved using the proposed controller where active load dynamics become relatively fast and smooth. The stability of the system is increased and also possible choices for controller parameters to make the system stable are wider than that the method suggested by N. Bottrell et a ${ }^{17-18}$.

This paper will cover two main parts: First part describes the proposed modified controller. The last part is the conclusion of the work.

\footnotetext{
* Author for correspondence
} 


\section{Proposed Modified AC Current Controller}

A comprehensive diagram of an active load is shown in Figure 1 Where it includes a LCL filter, DC voltage controller, AC current controller, switching bridge and DC load.

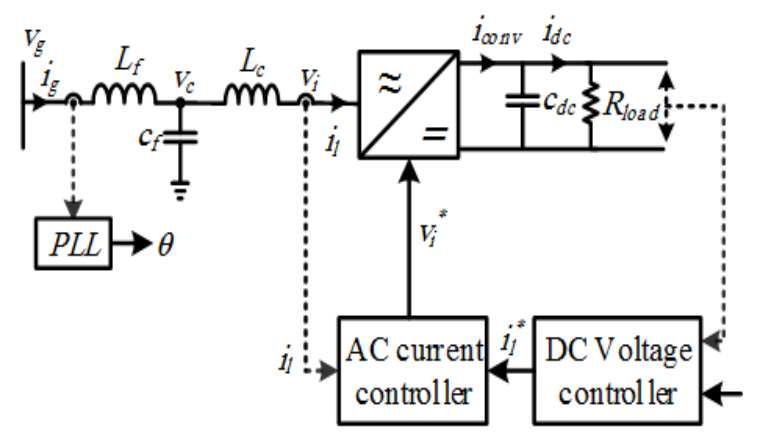

Figure 1. Active load subsystems.

The AC current controller of the active load is shown in Figure 2 where the current differences are the inputs of PI controllers. In opposite direction to an inverter, a positive current is defined as flowing from the grid connection to the switching bridge in the active load. Therefore, the current controllers of the inverter have been used in the case of the active load with a minus for the current differences as can be seen in Figure 2.

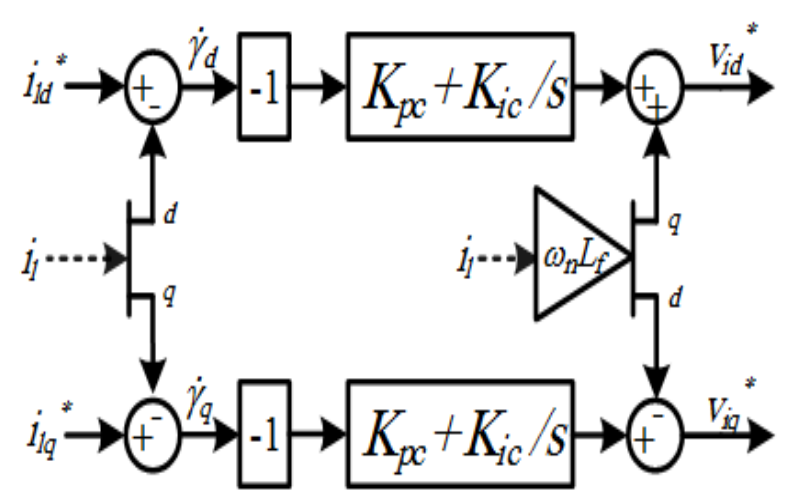

Figure 2. AC current controller of the active load.

The minus used for the current differences is not applied for the section after the PI controllers therefore it is modified as:

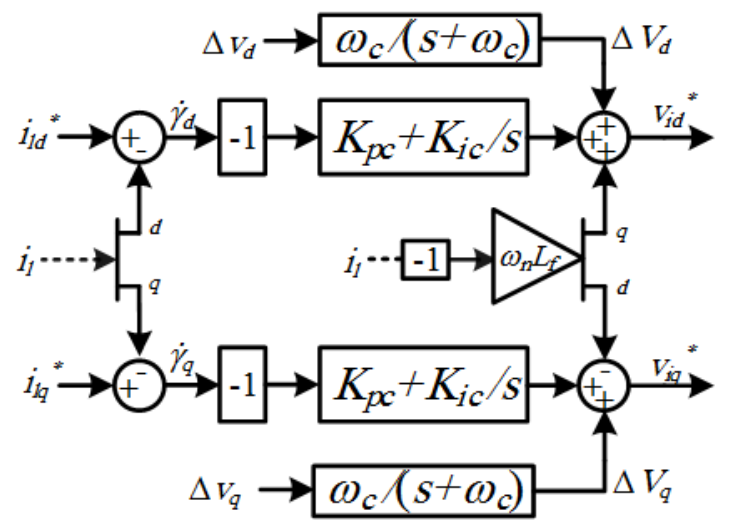

Figure 3. AC current controller of the active load.

As can be seen in Figure 3, two main modifications are applied as:

- As mentioned before, $i_{l}$ has an opposite direction compared to the inverter so each use of it must be with a minus sign. Therefore, $i_{1}$ is applied with a minus sign to combine with the PI controller outputs.

- LCL filter voltage drops are involved in thecalculation of the reference voltage so the reference voltage is obtained as:

$$
\begin{gathered}
v_{i d}{ }^{*}=-K_{p c}\left(i_{l d}{ }^{*}-i_{l d}\right)-K_{i c} \gamma_{d}-\omega_{n} L_{f} i_{l q}+\Delta V_{d} \\
v_{i q}{ }^{*}=-K_{p c}\left(i_{l q}{ }^{*}-i_{l q}\right)-K_{i c} \gamma_{q}+\omega_{n} L_{f} i_{l d}+\Delta V_{q}
\end{gathered}
$$

This modification has three advantages: First the final value of the switching voltage is reached faster than the conventional methods and therefore the transient time is limited to a shorter time. The second advantage is that the impedance of the LCL filter is involved in the control process especially its resistance. The third one is that it makes the selection of controller parameters easier and, as will be shown in the test results, a wider range of them can stable the system.

\section{Small Signal Model of the Proposed Method}

As the AC current controller of the active load is modified, the small signal model of the AC current controller presented by $\mathrm{N}$. Bottrell et al ${ }^{17-18}$ must be corrected. Therefore, based on (1) and Figure 3, the modified AC current controller small signal is obtained as: 


$$
\begin{aligned}
& \frac{d}{d t}\left[\begin{array}{l}
\Delta \gamma_{d q} \\
\Delta V_{d q}
\end{array}\right]=A_{1}\left[\begin{array}{l}
\Delta \gamma_{d q} \\
\Delta V_{d q}
\end{array}\right]+A_{2}\left[\begin{array}{c}
\Delta i_{d q}{ }^{*}
\end{array}\right]+A_{3}\left[\begin{array}{llll}
\Delta i_{l d q} & \Delta v_{c d q} & \Delta i_{g d q} & \Delta v_{g d q}
\end{array}\right]^{T} \\
& {\left[\begin{array}{c}
\Delta v_{i d q}^{*}
\end{array}\right]=B_{1}\left[\begin{array}{l}
\Delta \gamma_{d q} \\
\Delta V_{d q}
\end{array}\right]+B_{2}\left[\begin{array}{c}
\Delta i_{d q}{ }^{*}
\end{array}\right]+B_{3}\left[\begin{array}{llll}
\Delta i_{l d q} & \Delta v_{c d q} & \Delta i_{g d q} & \Delta v_{g d q}
\end{array}\right]^{T}}
\end{aligned}
$$

where

$$
\begin{aligned}
& A_{1}=\left[\begin{array}{ll}
0 & 0 \\
0 & a_{1}
\end{array}\right] \quad A_{2}=\left[\begin{array}{ll}
1 & 0 \\
0 & 1
\end{array}\right] \quad A_{3}=\left[\begin{array}{cc}
a_{2} & 0 \\
a_{3} & a_{4}
\end{array}\right] \quad a_{1}=\left[\begin{array}{cc}
-\omega_{c} & 0 \\
0 & -\omega_{c}
\end{array}\right] \\
& a_{2}=\left[\begin{array}{cccccc}
-1 & 0 & 0 & 0 & 0 & 0 \\
0 & -1 & 0 & 0 & 0 & 0
\end{array}\right] \quad a_{3}=\left[\begin{array}{cccccc}
0 & 0 & -\omega_{c} & 0 & 0 & 0 \\
0 & 0 & 0 & -\omega_{c} & 0 & 0
\end{array}\right] \\
& a_{4}=\left[\begin{array}{cc}
\omega_{c} & 0 \\
0 & \omega_{c}
\end{array}\right] \quad B_{1}=\left[\begin{array}{cccc}
-k_{i c} & 0 & 1 & 0 \\
0 & -k_{i c} & 0 & 1
\end{array}\right] \quad B_{2}=\left[\begin{array}{cc}
-k_{p c} & 0 \\
0 & -k_{p c}
\end{array}\right] \\
& B_{3}=\left[\begin{array}{cccccccc}
k_{p c} & -\omega_{n} L_{f} & 0 & 0 & 0 & 0 & 0 & 0 \\
\omega_{n} L_{f} & k_{p c} & 0 & 0 & 0 & 0 & 0 & 0
\end{array}\right]
\end{aligned}
$$

The small signal models of other sections of the active load are the same as the models presented by N. Bottrell et $\mathrm{al}^{17-18}$.

\section{Test Results}

The proposed modified control method is verified either in single mode operation of an active load or combined operation of it with a MG. In single mode operation, the active load is connected to an ideal voltage source while, in combined operation, the active load is connected to a MG. The MG is a $220 \mathrm{~V}$ (per phase RMS), $50 \mathrm{~Hz}$ prototype $\mathrm{MG}$ and built as shown in Figure 4. A resistive

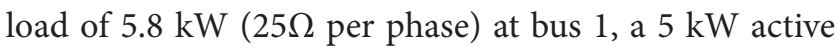
load at bus 3 and three inverters of equal rating (10 kVA) are considered in this real MG.

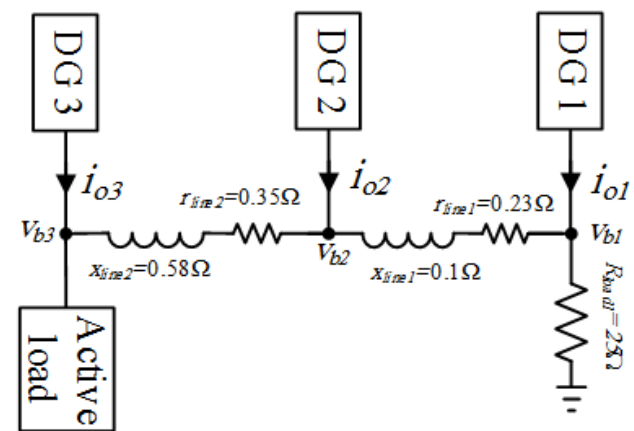

Figure 4. Combined operation of the active load when it is connected to a MG including three inverters.
To verify the proposed method, three tests are arranged as:

Test 1: In single mode, a disturbance including a $5-\mathrm{kW}$ step change of the active load is conducted while the AC current controller parameters are set too low.

Test 2: $\mathrm{R}_{\mathrm{dc}}$ is decreased down to the point where the stability is no longer attained for less than it and normal values are selected for the AC current controller parameters in single mode.

Test 3: In combined mode, $\mathrm{R}_{\mathrm{dc}}$ is decreased down to the point where the stability is no longer attained for less than it and normal values are selected for the AC current controller parameters.

Test 4: Kic is gradually increased while the minimum of $K p c$ needed to stable the system is obtained, in combined mode.

It should be noted that in Figures 5 through 11 the responses which is entitled "conventional method" is the response of the method suggested by $\mathrm{N}$. Bottrell et al ${ }^{17-18}$.

Table 1 gives effective eigenvalues of the active load in Test 1. It is obviously can be seen that two main oscillating poles of the AC current controller are damped where they are shifted from -9 to -34 .

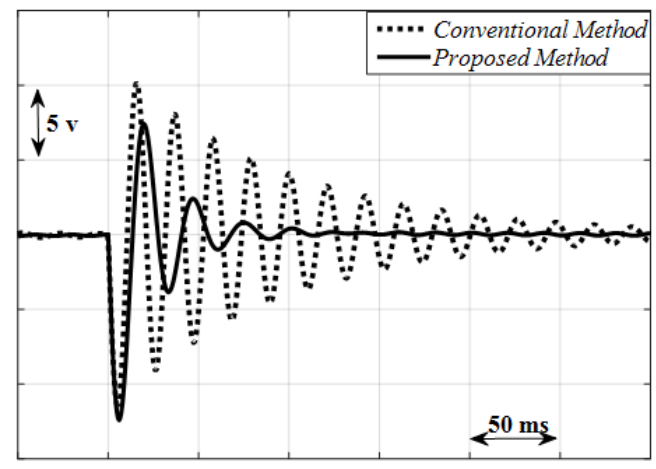

Figure 5. DC voltage of the active load in Test 1.

Table 1. Real parts of effective eigenvalues in Test 1

Poles Proposed Method Conventional Method

$\lambda_{1}$

$\lambda_{2}$

$-629.98$

$-654.78$

$-629.98$

$-654.78$

$\lambda_{3}$

$\lambda_{4}$

$\lambda_{5}$

$\lambda_{6}$

$-123.79$

$-122.60$

$-123.79$

$-122.60$

$-111.48$

$-112.92$

$-111.48$

$-112.92$

$-34.30$

$-9.27$

$-34.30$

$-9.27$

$-5.03$

$-5.00$

$-4.48$

$-4.45$ 
This improve can be clearly seen in Figures 5 and 6 where DC voltage and AC-grid current of the active load are shown. In Figure 5, the DC voltage of the active load has a great notch in the two methods and after the notch the response of the proposed method is damped in a short time but the conventional method response oscillate in a longer time. It is due to that the mentioned poles named $\lambda_{7}$ and $\lambda_{8}$ in Table 1 which are damped in the modified control method. In Figure 6, the same interpretation can be expressed for the AC-grid current (d-axis), $\mathrm{I}_{\mathrm{ld}}$, of the active load. This oscillating pole is of $47.4 \mathrm{~Hz}$ frequency.

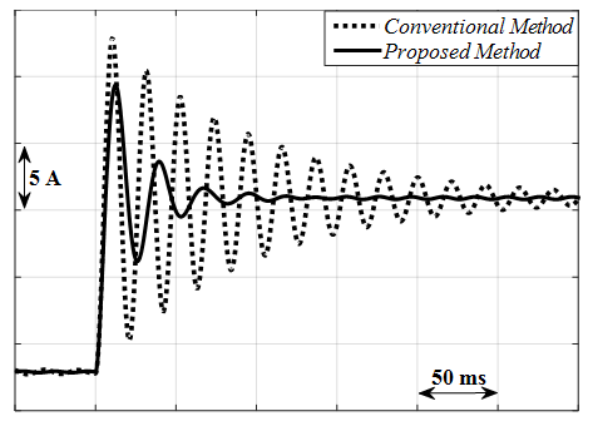

Figure 6. Active load AC-grid current, $I_{l d}$ in Test 1.

In Test 2, a severe condition for the active load controllers is investigated where the system is marginally stable. Figure 7 shows the DC voltage of the active load where high frequency oscillations can be seen in the responses. As can be seen in the figure, the oscillations are limited to one period of time in the proposed method while in the conventional method these oscillations are occurred in two different periods of time and extend the transient time about two times more than the proposed method. Active load AC-grid current, $\mathrm{I}_{\mathrm{ld}}$ in this test is shown in Figure 8. Similar to the DC voltage response, the current oscillations has limited to one period and lasted for a shorter time in the proposed method. It is clearly observed that the proposed method shows superior performance for severe conditions and helps the system to be stable in a shorter time and with small oscillations.

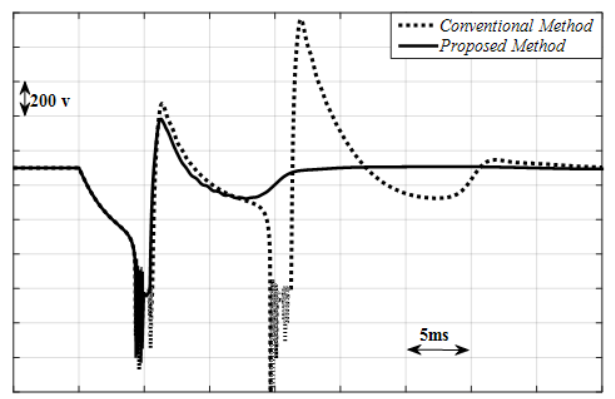

Figure 7. DC voltage of the active load in Test 2.

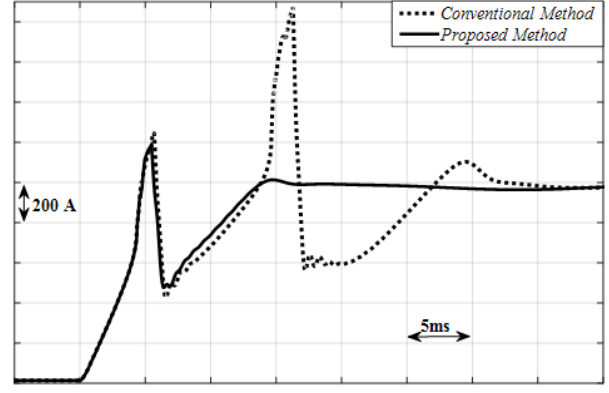

Figure 8. Active load AC-grid current, $\mathrm{I}_{\mathrm{ld}}$, in Test 2.

When the active load is connected to a MG, it follows dynamic of the MG. In this combined mode, active load controller settings must be selected of a more limited range than the single mode to stable the whole system. Figure 9 depicts the DC voltage of the active load in Test 3. As can be seen in the figure, the two methods present very small oscillation compared to Test 2 and it is due to that the MG limits oscillations of the active load and as mentioned limited choices for controller parameters. Figure 10 gives the AC-grid current, $\mathrm{I}_{\mathrm{ld}^{\prime}}$ of the active load and, as can be seen, presents similar responses as the DC voltage response. It is obvious that the proposed method has also better performance than the conventional method in this test.

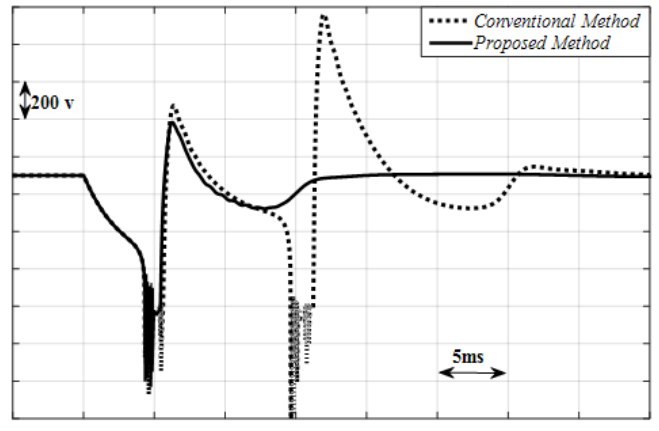

Figure 9. DC voltage of the active load in Test 3.

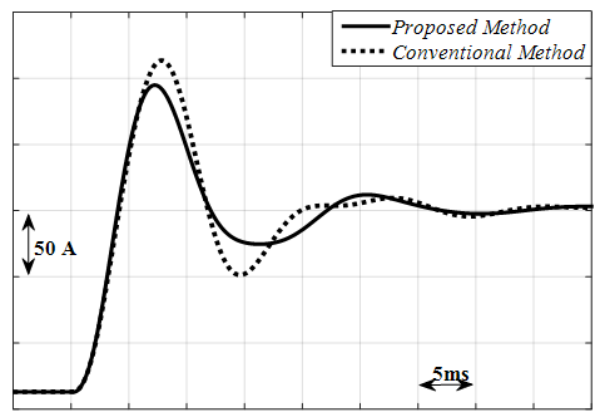

Figure 10. Active load AC-grid current, $I_{l d}$ in Test 3.3. 
Table 2 shows effective eigenvalues of the system in this test. Although most of these eigenvalues related to the inverters but, as can be seen, the proposed method presents more stable eigenvalues especially $\lambda_{4}$ and $\lambda_{5}$ related to the AC current controller of the active load.

Table 2. Real parts of effective eigenvalues in Test 3

\begin{tabular}{ccc}
\hline Poles & Proposed Method & Conventional Method \\
\hline$\lambda_{1}$ & -72.98 & -72.92 \\
$\lambda_{2}$ & -72.98 & -72.92 \\
$\lambda_{3}$ & -58.16 & -58.09 \\
$\lambda_{4}$ & -60.49 & -54.13 \\
$\lambda_{5}$ & -60.49 & -54.13 \\
$\lambda_{6}$ & -31.76 & -31.54 \\
$\lambda_{7}$ & -31.39 & -31.29 \\
$\lambda_{8}$ & -14.17 & -14.06 \\
$\lambda_{9}$ & -14.17 & -14.06 \\
$\lambda_{10}$ & -8.86 & -8.80 \\
$\lambda_{11}$ & -8.86 & -8.80 \\
\hline
\end{tabular}

In Test 4 , the system stability is investigated according to AC current controller parameters where Kiv is increased up gradually while minimum Kpa needed to stable the system is obtained. As can be seen in Figure 11 , the smaller amount of $K p a$ in the proposed method is needed to make the system stable for each specified amount of Kiv. It shows that the modified AC controller makes the system more stable even in normal conditions and also there are more choices for controller parameters.

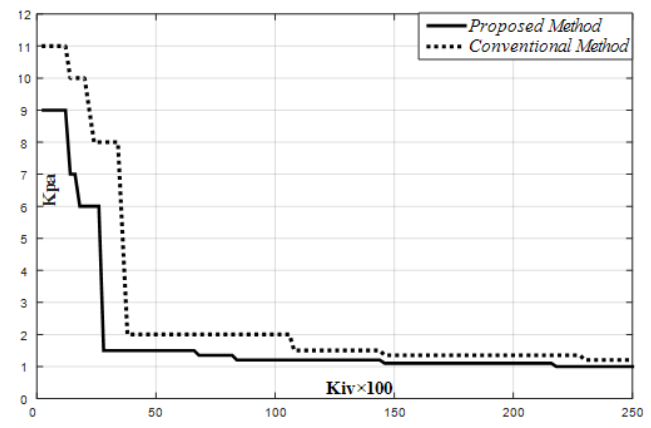

Figure 11. Minimum Kpa according to Kiv needed to stable the test system.

\section{Conclusion}

In this paper, a modified $\mathrm{AC}$ current controller for active loads has been presented. The conventional AC current controller has been improved using some corrections and adding new items to the control process. The proposed modified controller made active loads more stable especially in the case of severe conditions. The proposed method showed better performance in the case of not only single but also combined operation of active loads with MGs and there was a wider range to select more reliable controller parameters in the proposed method.

\section{References}

1. Sadeghi M, Gholami M. Fuzzy logic approach in controlling the grid interactive inverters of wind turbines. Indian Journal of Science and Technology. 2014 Aug; 7(8):1196-200.

2. Nayagam VS. Power reliability improvement of inverter with photovoltaic system. Indian Journal of Science and Technology. 2015 Mar; 8(6):570-3.

3. Sadeghi M, Gholami M. Optimized control strategy to adjust the intelligent universal transformer for integrating distributed resources to grid. Indian Journal of Science and Technology. 2014 Sep; 7(9):1260-6.

4. Manjula HS, Sasikumar M. Current harmonics reduction using Hysteresis Current Controller (HCC) for a wind driven self-excited induction generator drives. Indian Journal of Science and Technology. 2015 Jul; 8(13):1-6.

5. Kent MH, Schmus WR, Mccrackin FA, Wheeler LM. Dynamic modeling of loads in stability studies. IEEE Transactions on Power Apparatus and Systems. 1969 May; 88(5):756-63.

6. Kyriakides E, Farmer RG. Modeling of damping for power system stability analysis. Electric Power Components and Systems. 2004 Aug; 32(8):827-37.

7. Kwasinski A, Onwuchekwa C. Dynamic behavior and stabilization of DC microgrids with instantaneous constant-power loads. IEEE Transactions on Power Electronics. 2011 Mar; 26(3):822-34.

8. Cespedes M, Xing L, Sun J. Constant-power load system stabilization by passive damping. IEEE Transactions on Power Electronics. 2011 Jul; 26(7):1832-6.

9. Marx D, Magne P, Nahid-Mobarakeh B, Pierfederici S, Davat B. Large signal stability analysis tools in DC power systems with constant power loads and variable power loads: A review. IEEE Transactions on Power Electronics. 2012 Apr; 27(4):1773-87.

10. Rajesh M, Singh B. Single-phase boost rectifier fed SRM drive. IETE Journal of Research. 2014; 60(1):12-9.

11. Ariyasinghe D, Vilathgamuwa D. Stability analysis of microgrids with constant power loads. Sustainable Energy Technologies. IEEE International Conference on ICSET; Singapore. 2008 Nov. p. 279-84.

12. Emadi A, Khaligh A, Rivetta C, Williamson G. Constant power loads and negative impedance instability in automotive systems: Definition, modeling, stability, and control of 
power electronic converters and motor drives. IEEE Transactions on Vehicular Technology. 2006 Jul; 55(4):1112-25.

13. Yuvarajan S, Khoei A. A novel PWM for harmonic reduction and its application to ac-dc converters. IETE Journal of Research. 2002; 48(2):85-92.

14. Rahimi A, Emadi A. Active damping in dc/dc power electronic converters: A novel method to overcome the problems of constant powerloads. IEEE Transactions on Industrial Electronics. 2009 May; 56(5):1428-39.

15. Emadi A. Modelling of power electronic loads in AC distribution systems using the generalized state-space averaging method. Industrial Electronics Society. 2001. The 27th Annual Conference of the IEEE on IECON'01; Denver. 2001. p. 1008-14.

16. Sanders JA, Verhulst F. Averaging methods in nonlinear dynamical systems. Springer-Verlag; 1985.

17. Bottrell N, Prodanovic M, Green TC. Dynamic stability of a microgrid with an active load. IEEE Trans on Power Electronics. 2013; 28(11):5107-19.
18. Bottrell N, Green TC. Modeling microgrids with active loads. IEEE 13th Workshop on Control and Modeling for Power Electronics (COMPEL); Kyoto. 2012 Jun. p. 1-8.

19. Dannehl J, Fuchs FW, Hansen S. PWM rectifier with LCL-filter using different current control structures. European Conference on Power Electronics and Applications; Aalborg. 2007 Sep. p. 1-10.

20. Blasko V, Kaura V. A novel control to actively damp resonance in input LC filter of a three-phase voltage source converter. IEEE Transactions on Industry Applications. 1997 May; 33(2):542-50.

21. Rabinovici R, Avital M, Dagan KJ. An equivalent model for single and three phase power rectifiers with active loads. IEEE 27th Convention of Electrical \& Electronics Engineers in Israel (IEEEI); Eilat. 2012 Nov. p. 1-5 